\title{
RESIDUAL STRESS MEASUREMENT USING THE HOLE DRILLING TECHNIQUE ON COMPONENTS OUTSIDE THE ASTM E837 STANDARD
}

\author{
Yann Serra ${ }^{1, a}$, Xavier Ficquet ${ }^{2, b^{*}}$ and Ed Kingston ${ }^{2, c}$ \\ ${ }^{1}$ ENSIAME, Valenciennes, France \\ ${ }^{2}$ VEQTER, Bristol, UK \\ aserra.yn@gmail.com, ’xavier.ficquet@veqter.co.uk, ced.kingston@veqter.co.uk
}

Keywords: Hole drilling, Residual stress measurement, Numerical simulation

\begin{abstract}
The hole drilling technique is probably the most widely used residual stress measurement technique. The ASTM E837 standard covers hole drilling measurements for thin and thick specimens. VEQTER have encountered several cases when the specimen was between the thick and thin specification. In order to gain a greater understanding of the sensitivity of the analyses and accurately measure the residual stresses using the hole drilling technique within intermediate thickness specimens and within thin specimens containing non-uniform residual stresses a study was performed.
\end{abstract}

\section{Introduction}

The ASTM E837 standard describes the process from the attachment of the strain gauge rosette, through drilling of the hole to relieve the residual stresses, to the determination of the original residual stresses from the measured strains. The standard covers hole drilling measurements for thin specimens, i.e. those not exceeding a thickness of 0.4 times the diameter of the strain gauge circle (0.4D), or thick specimens greater than 1.2D [1]. For thin specimens, the standard restricts analysis to a uniform stress assumption, whereas for thick specimens the standard allows for both uniform and non-uniform stress assumptions. VEQTER have encountered several cases where the specimen was between the thick and thin specification or where a uniform stress assumption cannot be applied to a thin specimen. In order to gain a greater understanding of the sensitivity of the analyses and accurately measure the residual stresses using the hole drilling technique (Integral Method) within intermediate thickness specimens containing non-uniform residual stresses a study was performed.

This paper presents the finding of this study. First, several finite element models using different thickness ratios were created to determine a new set of calibration coefficients for the calculation of the residual stresses from the measured strains. This first phase highlighted the difficulties encountered and sensitivities in the analysis when using this technique for different thickness ratios. Several sets of calibration coefficients were calculated according to the thickness ratios. This paper then presents a numerical verification of the new set of calibration coefficients.

The finite element analyses were then verified against measurements within two four-point, plastically bent beam specimens with thickness ratios of about $0.5 \mathrm{D}, 1 \mathrm{D}$ and $2 \mathrm{D}$. The results were found to be in good agreement with the analytical and finite element predictions. 


\section{Determination of the residual stress using the hole-drilling technique}

The hole drilling technique is based on the measurement of the change in surface strain caused by the relief of the residual stresses during the machining of a shallow hole in a component. The principle is that the removal of stressed material results in the surrounding material readjusting its stress state to re-attain stress equilibrium. The measured surface strains allow for the back calculation of the existing residual stresses using a set of calibration coefficients $\bar{a}$ and $\bar{b}$. The formulae and calculations derived for the back calculation process were developed from a combination of experimental and finite element analyses for an infinite plate. The calibration constants are tabulated in the ASTM standard for thick and thin workpieces or can be calculated using numerical simulation [1,2]. The ASTM standard provides the specifications for the calculation of the residual stress for uniform or non-uniform stress field.

Table 1 Hole drilling specifications covered by the ASTM 837

\begin{tabular}{|c|c|c|}
\hline Thickness Ratio (Z) & Uniform Stress Field & Non-uniform Stress Field \\
\hline Thin: $Z<0.4 \mathrm{D}$ & $\begin{array}{c}\text { Through hole } \\
\text { Hole drilled all at once } \\
\bar{a} \text { and } \bar{b} \text { are scalar }\end{array}$ & Not covered by ATSM E837 \\
\hline Intermediate: $0.4 \mathrm{D}<\mathrm{Z}<1.2 \mathrm{D}$ & Not covered by ATSM E837 & $\begin{array}{c}\text { Case studied } \\
\text { Not covered by ATSM E837 }\end{array}$ \\
\hline Thick: $\begin{aligned} \mathrm{Z}<1.2 \mathrm{D} \\
\mathrm{D}\end{aligned}$ & $\begin{array}{l}\text { Blind Hole } \\
\text { Incremental hole drilling } \\
\bar{a}_{i} \text { and } \bar{b}_{i} \text { are vectors }\end{array}$ & $\begin{array}{c}\text { Blind Hole } \\
\text { Incremental hole drilling } \\
\bar{a}_{i j \text { and } \bar{b}_{i j} \text { are matrices }}\end{array}$ \\
\hline
\end{tabular}

The calculation of the residual stress profile from the measured strains is determined for uniform or non-uniform stress field at the exception of the uniform calculation on thick components by solving the matrix equations:

$$
\bar{a} P=\frac{E}{1+v} p, \quad \bar{b} Q=E q, \quad \bar{b} T=E t
$$

Where $\bar{a}$ and $\bar{b}$ are the calibration coefficients and $P, Q, T, p, q$ and $t$, are the stress and strain transformation variables which are defined by as following:

$$
\begin{array}{llc}
\mathrm{P}=\left(\sigma_{3}+\sigma_{1}\right) / 2 & \mathrm{Q}=\left(\sigma_{3}-\sigma_{1}\right) / 2 & T=\tau_{13} \\
p=\left(\varepsilon_{3}+\varepsilon_{1}\right) / 2 & q=\left(\varepsilon_{3}-\varepsilon_{1}\right) / 2 & t=\left(\varepsilon_{3}+\varepsilon_{1}-2 \varepsilon_{2}\right) / 2
\end{array}
$$

The dimensionless calibration coefficients $\bar{a}$ and $\bar{b}$ can either be in a form of a matrices, a vector or a scalar. They do not depend on the material properties and only vary with the type of the strain gauge (Type A, B or C), the size of the strain gauge and the hole geometry (hole radius and hole depth).

\section{Calculation of the calibration coefficients for intermediate thicknesses}

The calibration coefficients are determined using finite element analysis. They are calculated by applying a known stress conditions around the hole at different depth. Fig. 1 shows the loading condition to calculate the coefficient $\bar{a}_{\mathbf{1 1}}$. The strains are then measured at the location where the strain gauges are applied, hence their dependence to the type of rosette, as shown in Fig. 1. More details about the calculation of the coefficients can be found in [4]. In this study the coefficients were calculated with a 2D axisymmetric model using ABAQUS v6.10. Cumulative coefficients $\left(\bar{A}_{i j}\right.$ and $\left.\bar{B}_{i j}\right)$ were calculated during the study which can be directly compared with previous work $[3,4] . \bar{a}_{i j}$ or $\bar{A}_{i j}$ can be converted using the following relation: 


$$
\bar{a}_{i j}=\left\{\begin{array}{cl}
\bar{A}_{i j}-\bar{A}_{i j-1} & \text { if } 2 \leq j \leq i \\
\bar{A}_{i j} & \text { if } j=1
\end{array}\right.
$$

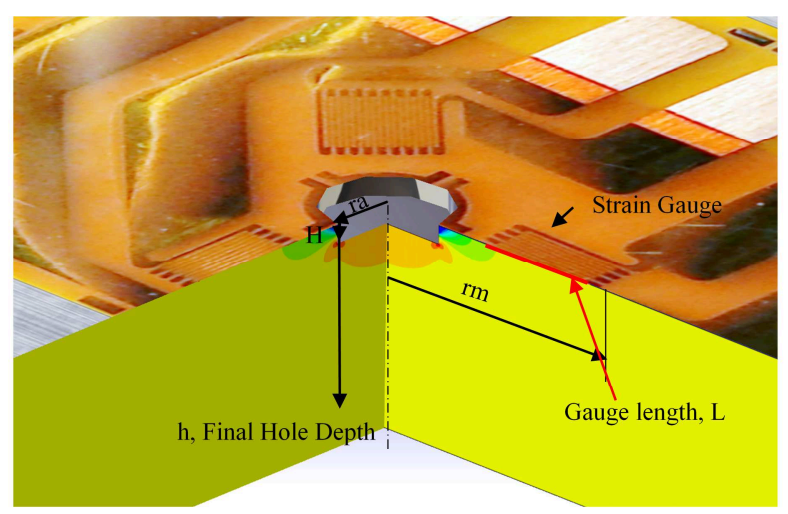

Fig. 1: Loading $\bar{a}_{11}$ condition showing strain gauge rosette type $\mathrm{A}$

For a comprehensive analysis of the whole "intermediate thickness" range, the coefficients were calculated with the thickness ratios of $0.4 \mathrm{D}, 0.6 \mathrm{D}, 0.8 \mathrm{D}, 1 \mathrm{D}, 1.2 \mathrm{D}, 1.4 \mathrm{D}$ as well as one thick ratio of $5 \mathrm{D}$ to compare the coefficients with those from the literature [3, 4]. For each thickness ratio, the calibration coefficients were calculated using hole ratios of $0.3 \mathrm{D}, 0.35 \mathrm{D}, 0.4 \mathrm{D} 0.45 \mathrm{D}$ and $0.5 \mathrm{D}$ as suggested by the ASTM standard. Each set of coefficients $\bar{a}$ and $\bar{b}$ for a given hole ratio and thickness ratio required 110 FEA models. A total of 3850 models were used in this study.

Fig. 2 shows only the cumulative calibration coefficients $\bar{A}_{i j}$ for different depth as the calibration coefficients for the shear stress $\bar{B}_{i j}$ present a similar profile. It can be seen that for thick workpiece the coefficients do not vary significantly which explains why the ASTM standard only use one set of coefficients. In the intermediate thickness range an important variation in the coefficients was observed. The set of calibration coefficients became dependent to the thickness ratio and needed to be calculated specifically as shown in the example in Fig. 3. The matrix is interpolated for a defined hole and thickness ratio from all the sets of intermediate calibration coefficients $\bar{a}$ and $\bar{b}$.

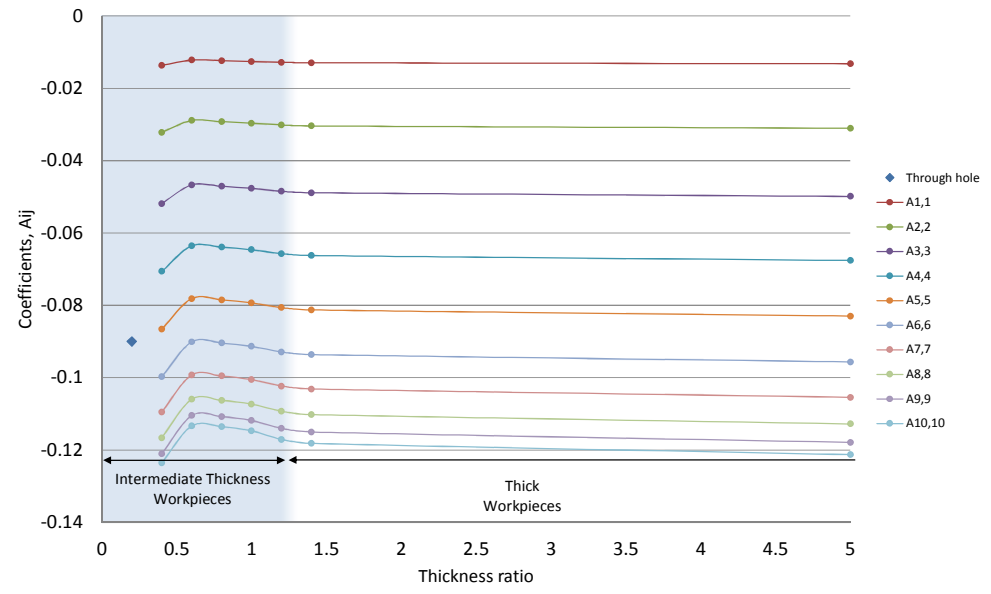

Fig. 2 Calibration coefficients $A_{i j}$ at different thickness ratio

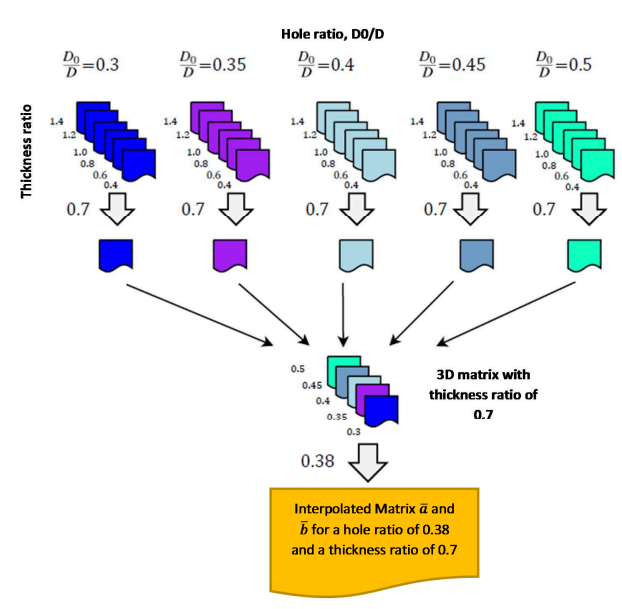

Fig. 3: Example of matrix $\bar{a}$ and $\bar{b}$ selection using a thickness of 0.7 and hole ratio of 0.38 


\section{Validation of the new intermediate calibration coefficients}

\section{Numerical validation}

To validate the new intermediate calibration coefficients a reverse analysis was performed using finite element analysis. The numerical validation was done with 2D and 3D models with an applied stress and an initial residual stress field respectively.

The 2D numerical validation was performed on an axisymmetric model with an applied pressure of $-200 \mathrm{MPa}$ as shown in Fig. 4, which introduced a uniform uniaxial stress field. The model had a thickness ratio of 0.8 and a hole ratio of 0.4 , which is in the middle of the intermediate thickness range. The hole drilling simulation was done in ten steps, each one corresponding to the drilling of one increment. For each step the strain was recorded at the surface and used to calculate the stresses. Fig. 5 shows a comparison of the stress calculated from the recorded strain using the new intermediate calibration coefficients and two different set of coefficients found in the literature [3, 4]. It can be seen that the calculation of the stress with the new intermediate coefficients are in excellent agreement with the applied stress.

The 3D numerical validation was performed with the same hole and thickness ratio as for the 2D validation. This time the stress was not applied, but was introduced as an uniaxial initial residual stress field using the ABAQUS subroutine SIGINI. A bending residual stress of $\pm 200 \mathrm{MPa}$ was introduced. Similarly to the $2 \mathrm{D}$ case, the hole drilling was simulated by incrementally removing the elements, with 10 increments of $0.12825 \mathrm{~mm}$ being removed. Fig. 5 shows the residual stress comparison with other set of calibration coefficients from the literature. For the 3D validation it can be seen that the new intermediate coefficients are in good agreement with the initial residual stress but do not provide better results than with the Bristol [3] or Schajer [4] coefficients set for thick specimen. This means that although the Bristol and Schajer coefficients were calculated for thick specimens, they can provide accurate results for those intermediate thickness.

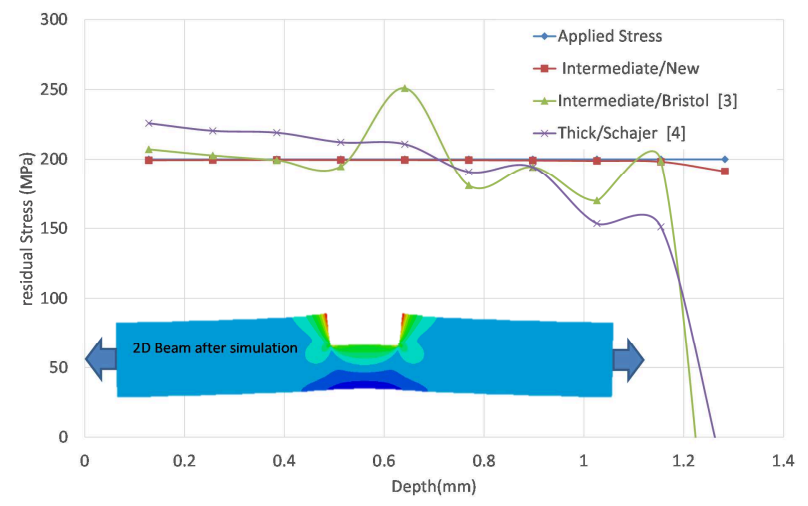

Fig. 4: Comparison of residual stress calculated on a 2D model using a thickness ratio of 0.8 and a hole ratio of 0.4

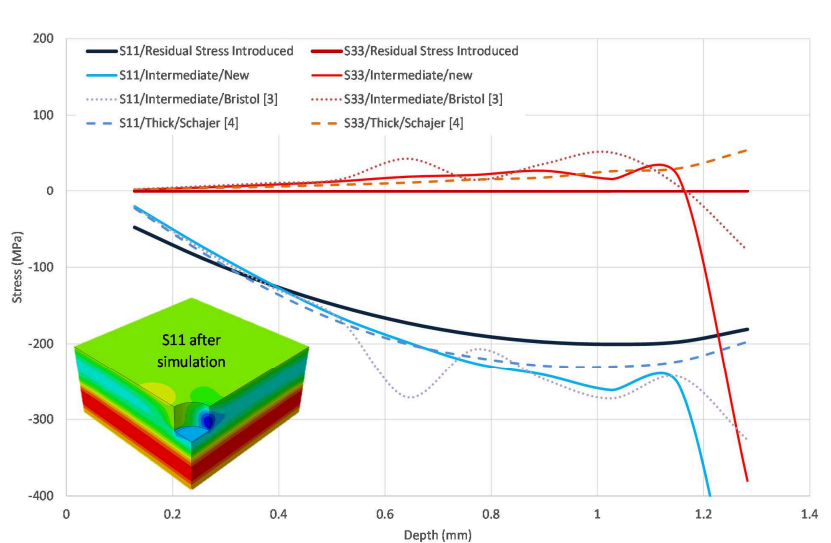

Fig. 5: Comparison of residual stress calculated on a $3 \mathrm{D}$ model using a thickness ratio of 0.8 and a hole ratio of 0.4

The differences between the calculated and initial residual stress were found to come mainly from the calibration coefficients for the shear stress $\bar{b}$, which explains why the $2 \mathrm{D}$ validation provide better results as the $\bar{b}$ coefficient is not used in this case. 


\section{Experimental Validation}

To experimentally validate the new intermediate coefficients, two aluminium beams were made. The first beam had dimensions of $24 \times 10 \times 200 \mathrm{~mm}$, the second beam had dimensions of $24 \times 6.35 \times 200 \mathrm{~mm}$. The beams were placed in a four point bending ring to introduce a known uniform bending residual stress in a zone large enough to attach two strain gauge rosettes [5]. On each beam a strain gauge of type CEA-062UL-120 and type CEA-125RE-120 (VISHAY ${ }^{\mathrm{tm}}$ ) was applied, giving for beam 1 a thick and intermediate thickness ratio, as summarised in Table 2. The strain gauge rosettes were applied before the loading as well as one uniaxial strain gauge to calculate precisely the residual stress introduced.

Table 2: Thickness ratio for beams 1 and 2

\begin{tabular}{|c|c|c|}
\hline $\begin{array}{c}\text { Strain gauge name } \\
\text { diameter (D) in mm) }\end{array}$ & $\begin{array}{c}\text { Beam } 1 \\
24 \times 10 \times 200 \mathrm{~mm}\end{array}$ & $\begin{array}{c}\text { Beam } 2 \\
24 \times 6.35 \times 200 \mathrm{~mm}\end{array}$ \\
\hline CEA-062UL-120 (5.13) & $1.95 \mathrm{D}$ (Thick) & $1.2 \mathrm{D}$ (Thick) \\
\hline CEA-125RE-120 (10.26) & $0.97 \mathrm{D}$ (Intermediate) & $0.62 \mathrm{D}$ (Intermediate) \\
\hline
\end{tabular}

Fig.6 and Fig.7 show photographs of beams 1 and 2 during the intermediate thickness measurement. The green marks show the limit of the uniform bending stress. The residual stresses were computed using the ASTM standard coefficients and the new intermediate coefficients from this research. The results from the beam 1 and beam 2 measurements are shown in Fig.6 and Fig. 7 respectively, compared with the predicted residual stress calculated from the measured deformation during four point bending.

The results of the two measurements calculated with the ASTM (Red) and the new intermediate coefficients (Blue) on the beam one and two can be seen respectively in Fig.6 and Fig.7. The new intermediate coefficients were all interpolated to the exact thickness ratio as shown in Fig.3. Fig.6 shows the results for a thickness ratio of 1.24D and 0.64D. The results have been compared with calculated results (Black) modelled using elastic plastic behaviour [5].

All results were in good agreement with the calculated residual stress, as well as the 0.64D measurement calculated with the ASTM coefficients which is in the middle of the $0.4 \mathrm{D}$ to $1.2 \mathrm{D}$ range and at the peak value of the coefficients $\bar{a}$ and $\bar{b}$ as shown in Fig.2.

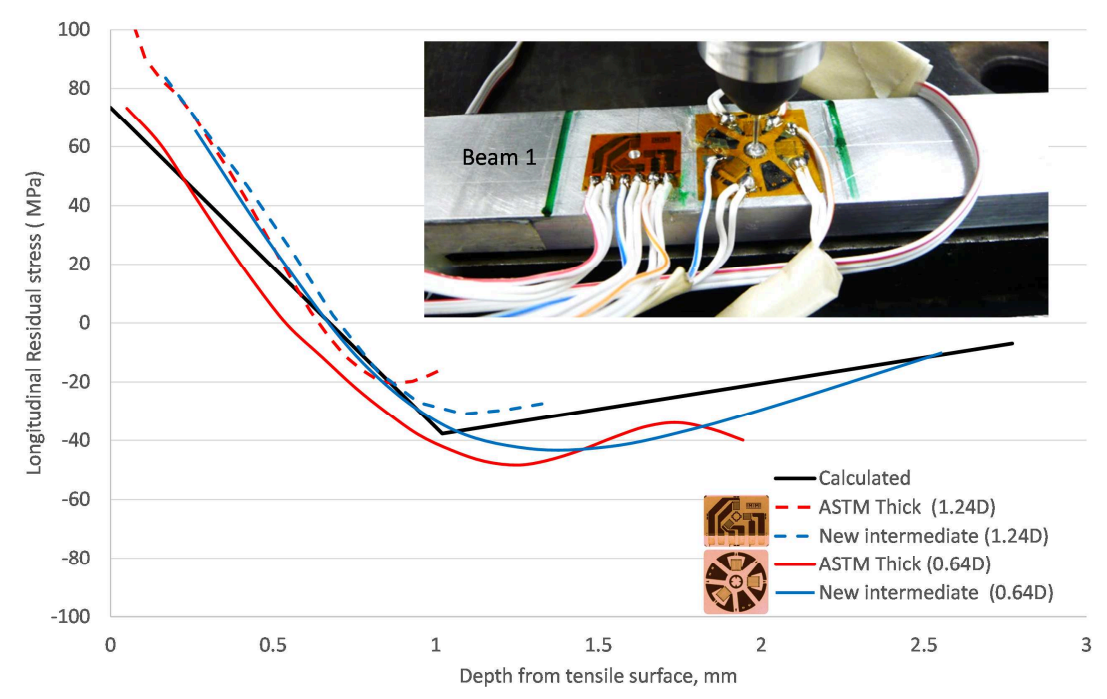

Fig.6: Comparison of the residual stress calculated with the new intermediate and ASTM coefficients on beam 1 at different thickness ratios 
The results for a thickness ratio of 0.97D and 1.97D are shown Fig.7. Again all results were in good agreement with the calculated residual stress.

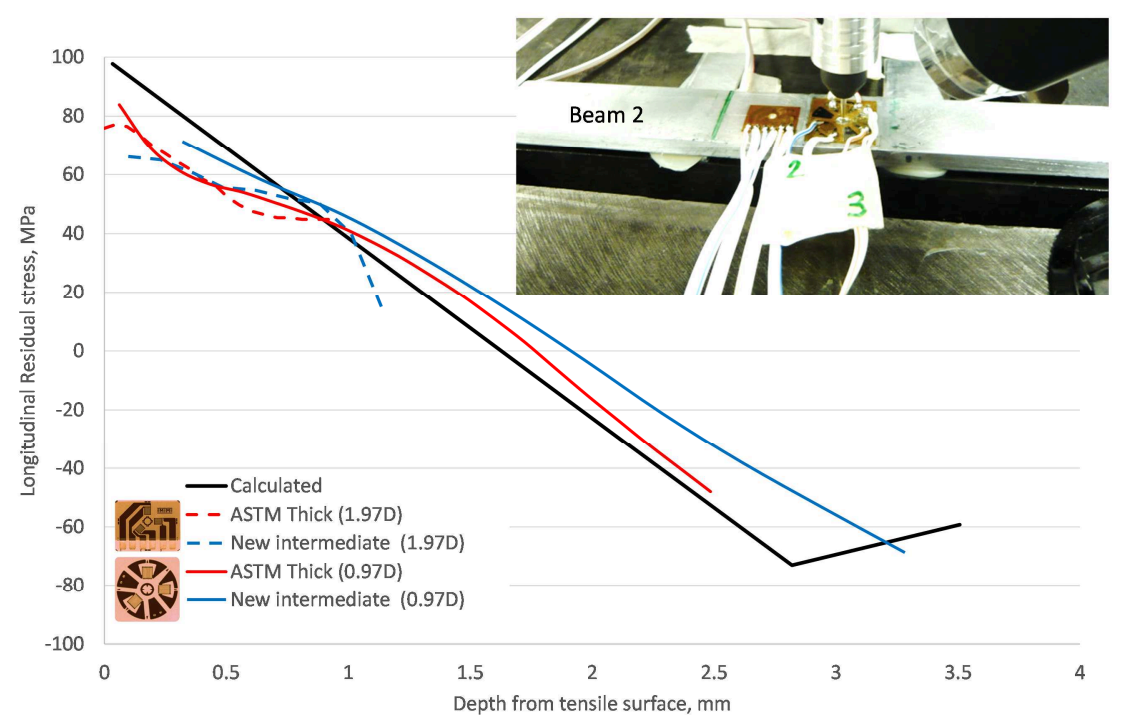

Fig.7: Comparison of the residual stress calculated with the new intermediate and ASTM coefficients on beam 2 at different thickness ratios

\section{Conclusion}

A new set of calibration coefficients was determined within a range of 0.4 to 1.2 times the diameter of the strain gauge rosette, not covered by the ASTM standard. A numerical and experimental validation was done. The results calculated with the new intermediate coefficients were all in good agreement with the predicted residual stress. The experimental results also gave some credit to the change in the ASTM standard to the updated version [2] which reduced the intermediate zone to $0.2 \mathrm{D}$ to $1 \mathrm{D}$.

\section{Bibliography}

[1] American Society for Testing and Materials. E837-08 Standard Test Method for Determining Residual Stresses by the Hole-Drilling Strain-Gage Method. (2008)

[2] American Society for Testing and Materials. E837-13a Standard Test Method for Determining Residual Stresses by the Hole-Drilling Strain-Gage Method. (2013)

[3] G. Holland, S. Lewis. Advances in Incremental Centre Hole Drilling. Bristol University, (2005)

[4] Measurement of non-uniform residual stresses using the hole-drilling method. Schajer, G.S., Journal of Engineering Material and Technology. (1988)

[5] D. Goudar. "Quantifying Uncertainty in Residual Stress Measurement using Hole Drilling Techniques". PhD thesis, Bristol University, (2011) 